\title{
Recognition of Power Quality Disturbances using Fuzzy Expert Systems
}

\author{
P.KANIRAJAN ${ }^{1}$, M.JOLY ${ }^{2}$ AND T. ESWARAN ${ }^{3}$ \\ Electrical and Electronics Engineering \\ ${ }^{1}$ Saveetha Engineering College and ${ }^{2}$ Saveetha Engineering College, ${ }^{3}$ AKT Memorial College of \\ Engineering and Technology, INDIA
}

\begin{abstract}
This paper presents a new approach to detect and classify power quality disturbances in the power system using Fuzzy C-means clustering, Fuzzy logic (FL) and Radial basis Function Neural Networks (RBFNN). Feature extracted through wavelet is used for training, after training, the obtained weight is used to classify the power quality problems in RBFNN, but it suffers from extensive computation and low convergence speed. Then to detect and classify the events, FL is proposed, the extracted characters are used to find out membership functions and fuzzy rules being determined from the power quality inherence. For the classification, 5 types of disturbance are taken in to account. The classification performance of FL is compared with RBFNN. The clustering analysis is used to group the data in to clusters to identifying the class of the data with Fuzzy $\mathrm{C}$-means algorithm. The classification accuracy of FL and Fuzzy C-means clustering is improved with the help of cognitive as well as the social behavior of particles along with fitness value using Particle swarm optimization (PSO),just by determining the ranges of the feature of the membership funtion for each rules to identify each disturbance specifically. The simulation result using Fuzzy C-means clustering possess significant improvements and gives classification results in less than a cycle when compared over other considered approach.
\end{abstract}

Key-Words: - Power Quality, Fuzzy C-means Clustering, Fuzzy Logic, Radial Basis Function Neural Network, Wavelet Transformation, Particle Swarm Optimization.

Received: May 4, 2020. Revised: September 30, 2020. Accepted: November 12, 2020. Published: December 1, 2020.

\section{Introduction}

The quality of electric power is more important because one of the main problem the industries facing is the distortion in electrical supply. The disturbance such as voltage sag, swell with and without harmonics, momentary interruption, harmonic distortion, notch, flicker, spike and transients causing problems such as malfunction, instability, short life times, failure of electrical equipment and so on. In power system, these disturbances need to be identified in order to improve power quality (PQ). PQ events identification is difficult because it involves wide range of disturbance categories. Therefore, the decision boundaries of disturbance features may overlap. For these reasons, the need of power quality analysis has been strongly increasing. Many techniques have been proposed in the literature to detect and classify the events envelope. Traditionally, probabilistic approach has been used for time varying signals in a power quality analysis, assuming that the power line disturbance components vary too slowly to affect the accuracy of analytical process [1],[2]. Another paper has suggested a combination of spectral method with probabilistic approach, also referred as evolutionary spectrum [3]. The Discrete Fourier Transforms (DFT), which is computed via the Fast Fourier Transforms (FFT), is used to extract the features in the waveforms [4]. However, the accuracy of the DFT algorithm is affected by the product available in the voltage waveform. Transient characteristics of disturbances waveforms are discussed in5, since they pertain to signal analysis. This analytic technique includes the Short-Time Fourier Transform (STFT) which briefs time-frequency information related to disturbance waveform. However, the disturbances signal cannot be adequately described in this transform, due to fixed window size[6].

For these reasons, S-Transform (ST) is often adopted as a tool for signal analysis. The superior properties of the ST are that the modulating sinusoidal is fixed with respect to the time axis, while the localizing scalable Gaussian window 
dilates and translates. As a result, the phase spectrum is absolute in the sense that it always referred to the origin of the time axis, the fixed reference point. ST is found to be superior [7], However, the computational time is very large compared to Wavelet Transform (WT), which is undesirable for on-line applications. WT based approach, such as wavelet Multi-resolution analysis (MRA), has been widely applied to solve these issues [8], [9].

Wavelet transform and MRA provide a short window for high frequency components and long window for low frequency components [10], [11] and hence, provides an excellent time frequency resolution. This allows WT for analysis of signals with localized disturbances components and also for classifying low and high frequency power quality problems, using the properties of WT technique and the features of the decomposed waveforms along with ANN algorithm [12],[13] it is possible to extract important information from a disturbance signal for to determine the type of disturbance. The energy of the distorted signal will be partitioned at different resolution levels in different ways depending on the event available. The standard deviation can be considered as a measure of energy signal with zero mean [14].

The classification of seven types of PQ disturbances with self-organizing learning array system considering 11 features, besides 22 families of wavelet are tested to identify the best one for a better classification. Classification of seven types of PQ events using wavelets and Probabilistic Neural Network (PNN) is done [15].Energy distribution at 13 decomposition levels of wavelet and time duration of each disturbance are taken as features and applied to PNN for classification. If large number of features is considered, it may result in high memory and computational overhead. Further, eleven types of PQ events are also classified with the help of ST and PNN using only fourdimensional feature sets for training and testing [16].

Considering all these issues related to detection and classification of PQ events, Fuzzy logic based on Wavelet Transform is proposed. The detection and classification also done with Fourier linear combiner along with fuzzy system which extracts the amplitude and phase of the fundamentals signal ,but in this computational burden is very high [17].In this fuzzy expert system is used to detect the disturbances, this method just uses voltage signal and they are decomposed with help of wavelet. In thisAmplitude, Slope, Standard Deviation, Variance, Skewness, Kurtosis, Shannon Entropy, Log Energy
Entropy, Norm Entropy along with Energy of each component of signal were obtained and used as a inputs to FL to distinguish events types using fuzzy logic tool box in MATLAB .To group and identify the disturbance a clustering analysis is used which will group the data in to clusters. For data clustering the well-known Fuzzy C-means algorithm is used commonly. In this paper, a Fuzzy C-Means Clustering along with PSO is used to cluster the features in to specific groups so as to identify and classify the nature of the disturbances.

From the simulation results, it is found that Fuzzy $\mathrm{C}$-means algorithm classifies the PQ event more effectively than the FL and RBFNN.The classification accuracy of Fuzzy C-means Clustering algorithm is improved, with the help of cognitive as well as the social behavior of particles along with a fitness value by PSO algorithm. PSO can be a solution which models the cognitive as well as the social behavior of a flock of birds which are in search of food over an area 18, 19.It improves Fuzzy C-meansclustering algorithmin various aspects mainly to specify the features of the membership functions used to identify the disturbances

Here, less number of features is required for effective classification of 5 types of PQ events. The Fuzzy C-means algorithm-PSO provides accurate results even with inputs found out under high noisy conditions. The performance results were compared with other considered approach, to prove the stability and accuracy of the classification. The proposed method is tested with the inclusion of white noise in the signal. From the simulation results, it is found that Fuzzy C-means Clustering algorithm-PSO classifies the PQ event more effectively than other well-known algorithms.

To summarize, first the work handles with Wavelet Transformation and feature extraction by WT needed by the neural networks, FL and Fuzzy Cmeans for effective classification for all the 5 types of disturbances. Next the paper describes the structure and results and discussion about detection and classification of PQ events using RBFNN and Fuzzy logic. Then the performance of Fuzzy Cmeans clustering algorithm were discussed, finally performance of FL and Fuzzy C-means algorithm with PSO is results were compared with other considered approach. 


\section{Wavelet Transforms}

Wavelet transformation has the ability to analyze different power quality disturbances in both time and frequency domain. The wavelet transform is useful in extracting features of various power quality disturbances. Wavelet analysis deals with expansion of function in terms of a set of basis function. However, wavelet analysis expands functions not in terms of trigonometric polynomials but in terms of wavelets. Moreover, another important property that the wavelet possesses is perfect reconstruction, which is the process of reassembling a decomposed signal or image into its original form without loss of information.

\subsection{Multi-resolution Analysis}

Scaling function and wavelet function are used as a building block to decompose and construct the signal at different resolution levels in MultiResolution Analysis (MRA).Representation of signals at various levels of resolution is the ultimate goal of MRA.MRA consists of two filters in each level and they are low pass and high pass filters. The resolution of the signal, which is a measure of the amount of detail information in the signal, is changed by the filtering operations, and the scale is changed by up-sampling and down-sampling operations. Down-sampling, a signal corresponds to reduction of the sampling rate, or removing some of the samples of the signal. On the other hand, upsampling a signal corresponds to rising of the sampling rate of a signal by adding new samples to the signal. MRA decomposition and reconstruction are shown in Figure.1 (a) and (b).

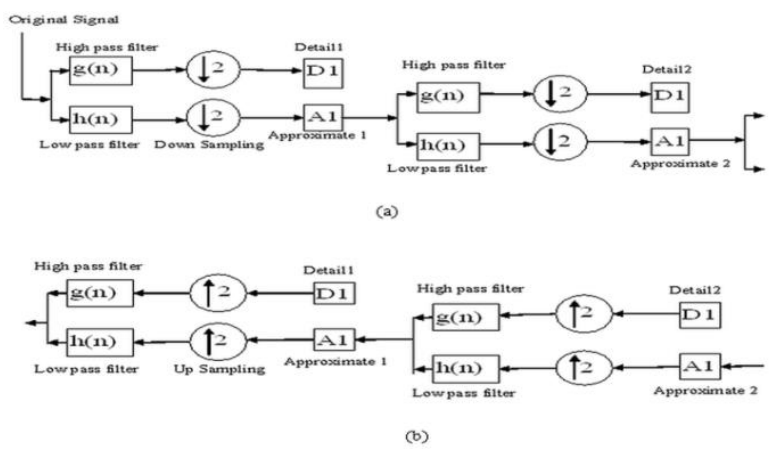

Fig.1.(a)Multi-resolution analysis decomposition and (b) Multi-resolution analysis reconstruction

Assume a signal $\mathrm{x}[\mathrm{n}]$, discrete time signal is distributed in 2 level. This signal is filtered into high frequency component in level 1 by using a high pass filter $(\mathrm{g}(\mathrm{n}))$ and low frequency components in level 2 by using a low pass filter $(\mathrm{h}(\mathrm{n}))$. This signal is passed through down sampling in MRA level 2.The components in level 1 are used as initial signals. These signals are passed through high-pass filter and low-pass filter. The outputs of filter can be mathematically expressed as in equation (1) and (2) as follows.

$$
\begin{aligned}
& y 1[k]=\sum_{n} x[n] \cdot g[2 k-n] \\
& y 2[k]=\sum_{n} x[n] \cdot h[2 k-n]
\end{aligned}
$$

Where $\mathrm{g}(\mathrm{n})$ is a high pass filter.

$h(n)$ is a low-pass filter.

Where $\mathrm{y} 1[\mathrm{k}]$ and $\mathrm{y} 2[\mathrm{k}]$ are the outputs of the highpass and low-pass filters, respectively.

\subsection{Wavelet based Feature Extraction}

Power system comprises of various kinds of electrical disturbances such as sag, swell, momentary interruption, voltage fluctuation, harmonics etc. and for the analysis they are generated using MATLAB code. The generated waveform shows the plot of amplitude of a givenmagnitude in the time frequency coordinates system for the following signals shown in Figure. 2 (a)-2(e).
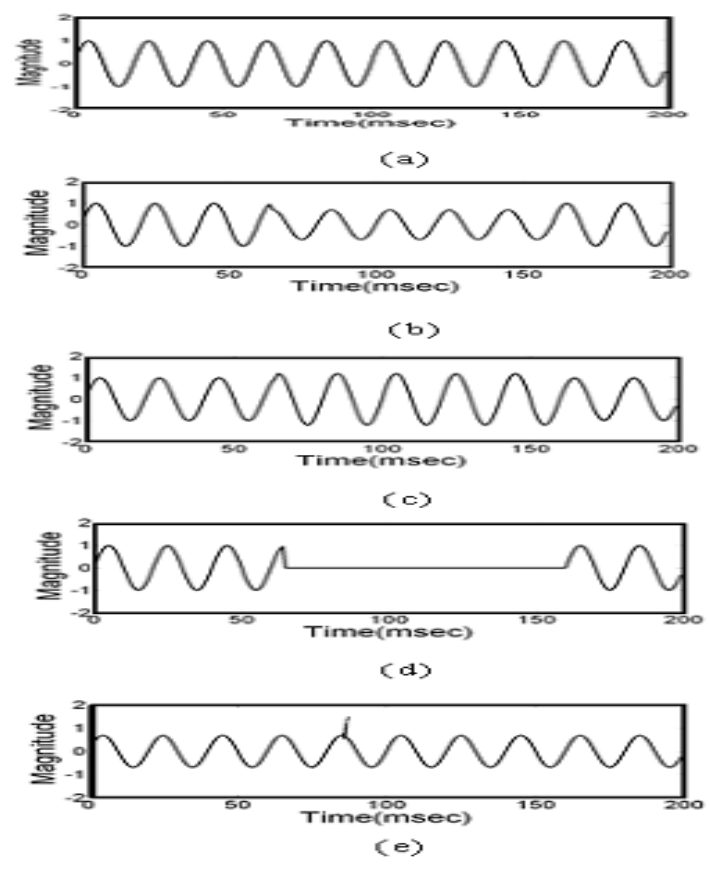

Fig.2. (a) Normal Signal,(b)Pure Sag,(c)Pure swell, (d)Momentary interruption, (e) Transients 
Which all are decomposed by wavelet to extract features with the appropriate selection of the wavelet and decomposition scale. The following signals $\mathrm{C} 1$ to $\mathrm{C} 5$ were used in the analysis.

C1-Normal Signal, C2-Pure Sag, C3 Pure swell, C4Momentary interruption and C5- Transients

\subsection{Selection of Wavelets and Decomposition Scale}

In this section, a simple yet effective method to detect and classify power quality disturbance, there are a number of basis functions that can be used for wavelet transformation. The wavelet function used in the transformation is through translation and scaling, it determines the characteristics of the resulting wavelet transform, Therefore, the details of the particular application should be taken in to account and the appropriate wavelet function should be chosen in order to use the wavelet transform effectively. The wavelets are chosen based on their shape and their ability to analyze the signal in a particular application. So the best wavelet function and optimal decomposition scale need to be carefully selected. Wavelet energy is the index to reflect the energy concentration of wavelet coefficients on certain scales. The larger the wavelet energy, the more the information is preserved after decomposition.

The definition of total energy and average power for a signal $x[n]$ being expressed as follows in equations (3)-(5).

$E=\sum_{n=-\infty}^{\infty} x^{2}[n]$

And the average power is

$P=\lim _{x \rightarrow \infty} \frac{1}{2 N} \sum_{n=-N}^{N} x^{n}[n]$

And for a periodic signal of fundamental period $N$, the average power is given by

$P=\frac{1}{N} \sum_{n=-0}^{N-1} x^{2}[n]$

In this Daubechies (Db) and Symlet (Sym) wavelets are taken for the further analysis. Thedaubechies wavelets are a family of orthogonal wavelet defining a discrete wavelet transform, characterized by a maximal number of vanishing moments and given support to each wavelet, and there is a scaling function which generates an orthogonal multiresolution analysis. Thesymlet's are nearly symmetrical wavelets proposed by daubechies as modification to the $\mathrm{Db}$ family, and the properties of these two wavelet families are similar. These wavelets have been chosen because they have shown best performance in analyzing disturbance signals. The wavelet corresponding to the highest total energy is chosen as the best wavelet function, and the scale corresponding to the highest energy is chosen as the optimal decomposition scale. Then all the proposed disturbance were taken in this paper and results are listed in Table 1 and Table 2, the elements shaded indicates the highest wavelet energy of a specific signal, corresponding to a certain wavelet functions. Among these $\mathrm{Db} 4$ have highest wavelet energy levels, and chosen as the best wavelet for feature extraction. Table 2 shows signal decomposition by $\mathrm{Db} 4$ in to scales and it is evident that the wavelet energy at scale 5 is the highest and can be used as the optimal decomposition scale for MRA.

The parameters of voltage waveforms during power quality events are statistically different from those that are calculated during an event free time period. In this work, features such asAmplitude, Slope, Energy, Standard deviation, Skewness, Kurtosis, Shannon Entropy, Log Energy Entropy and Norm Entropy of transformed signals are extracted and energy at each decomposition level, which has the ability to quantify the magnitude of variation within the signal, is also extracted. The extracted features help to distinguish one disturbance event from another. In order to extract feature of these signals, the standard deviation of power quality problem signal is subtracted from standard deviation of pure sinusoidal waveforms in case of analysis based on standard deviation multi-resolution analysis. In order to reduce the features dimension, the detail and approximate information for future training and testing will not be used directly. Instead, energy at each decomposition level is used as a new input variable for accurate and for faster classification. In this way, the wavelet based feature extraction for future analysis has been constructed.

\section{Radial Basis Function Neural Network}

Radial basis function neural network consists of a network similar to back propagation network as shown in Figure .3 with a single hidden layer. RBFNN proves to be best for classification task from the investigation result presented [20].

Each hidden layer consists of smoothing factor and centroids. The distance between the input and the centroid are normally computed by the neurons. 
Table 1. Results of Selection of Wavelet Function

\begin{tabular}{|l|c|c|c|c|c|c|c|c|}
\hline \multirow{2}{*}{} & \multicolumn{5}{|c|}{ Daubechies } & \multicolumn{5}{c|}{ Symlets } \\
\cline { 2 - 10 } & \multicolumn{3}{|c|}{ Level } & \multicolumn{5}{c|}{ Level } \\
\cline { 2 - 10 } Events & 2 & 3 & 4 & 5 & 2 & 3 & 4 & 5 \\
\hline C1 & 0.9907 & 0.9826 & 0.9921 & 0.9899 & 0.9819 & 0.9717 & 0.9846 & 0.9837 \\
\hline C2 & 0.9748 & 0.9601 & 0.9863 & 0.9726 & 0.8915 & 0.9256 & 0.9793 & 0.9614 \\
\hline C3 & 0.8417 & 0.8462 & 0.8917 & 0.8733 & 0.8367 & 0.8511 & 0.8845 & 0.8815 \\
\hline C4 & 0.8342 & 0.8172 & 0.8915 & 0.8678 & 0.7942 & 0.8173 & 0.8591 & 0.8498 \\
\hline C5 & 0.8941 & 0.9118 & 0.9218 & 0.9197 & 0.8762 & 0.8771 & 0.8924 & 0.8891 \\
\hline
\end{tabular}

Table 2. Results of Selection of Scale

\begin{tabular}{|l|c|c|c|c|c|c|}
\hline \multirow{2}{*}{ Events } & \multicolumn{7}{|c|}{ Daubechies } \\
\cline { 2 - 7 } & \multicolumn{7}{|c|}{ Scale } \\
\cline { 2 - 8 } & 1 & 2 & 3 & 4 & 5 & 6 \\
\hline \multirow{2}{*}{ C1 } & 0.3442 & 0.3639 & 0.4171 & 0.4987 & 0.5074 & 0.4982 \\
\hline C2 & 0.3911 & 0.3948 & 0.4794 & 0.5217 & 0.5737 & 0.4955 \\
\hline C3 & 0.3841 & 0.3979 & 0.4288 & 0.4812 & 0.4919 & 0.4871 \\
\hline \multirow{2}{*}{ C4 } & 0.04681 & 0.0517 & 0.0594 & 0.0634 & 0.0678 & 0.0646 \\
\hline C5 & 0.2941 & 0.2974 & 0.2987 & 0.3156 & 0.3417 & 0.3196 \\
\hline
\end{tabular}

The outputs are a radial symmetrical function of the distance [21].

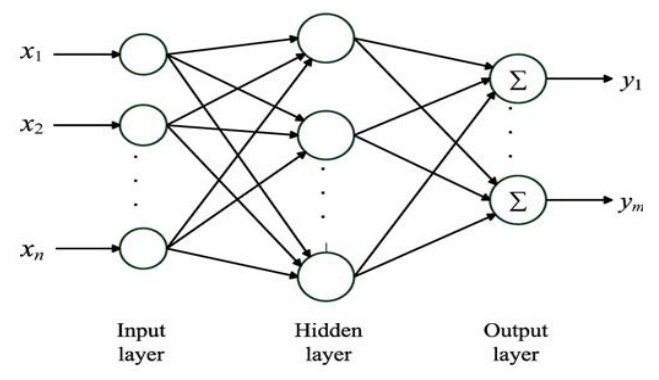

.Fig.3.Architecture of NN
When it is close to value the output will be a strong one.The real mapping function $f_{m}$ in general formis expressed in equation (6).

$$
f_{m}(x)=\sum_{i=1}^{M} w_{i} k\left[\left(x_{i}-c_{i}\right) / o_{i}(6)\right.
$$

The function $k$ is a radial symmetrical kernel function computed by $M$ kernel units.

The Gaussian exponential function used in RBF is expressed in equation (7).

$$
f(x)=\beta \exp \left(-\sum_{i}\left[\left(x_{i}-c_{i}\right) / o_{i}\right]^{2}\right)
$$

According to the training data set, centroid and constant have to be chosen. 


\section{Fuzzy C-Means Clustering Algorithm}

\section{Amplitude,Slope,Energy,Standard}

deviation,Variance ,Skewness,Kurtosis,Shannon Entropy,Log Energy Entropy, a and Norm Entropy have been extracted from the waveforms with the help of WT.After the extraction variance and normalized values are the most distinguished features much suited in the analysis done with Fuzzy C-means clustering to classify and group the distinct disturbance. In multi-dimensional space Fuzzy C-means algorithm groups the data points in to a specific number of cluster [17], [24].The extracted feature were given as a input to Fuzzy cmeansclustering .Determining of cluster center $c_{i}$ and the membership matrix $U$ are important which is done based on minimization of the objective function shown in equation (8).

$$
J_{m}=\sum_{i=1}^{N} \sum_{j=1}^{C} u_{i j}{ }^{m}\left\|x_{i}-c_{i}\right\|^{2}
$$

Where $u_{i j}$ is the degree of membership of $x_{i}$ in clusterj, $x_{i}$ is the $i^{\text {th }}$ of n-dimentional data, $m$ is the number of clusters, and $c_{i}$ is the n-dimensional centres of the cluster which is expressed in equation (9) and (10).

$C_{i}=\frac{\sum_{i=1}^{N} u_{i j}{ }^{m} x_{i}}{\sum_{i=1}^{N} u_{i j}{ }^{m}}$

$u_{i j}=\sum_{\mathrm{k}=1}^{\mathrm{c}}\left[\frac{\left\|x_{i}-c_{j}\right\|}{\left\|x_{i}-c_{k}\right\|}\right]^{\frac{-2}{\mathrm{~m}-1}}$

In Figure.4 the clustering using fuzzy c-means algorithm is shown. In which swell and remaining disturbance are were put in to two clusters and depicted by stars and circle.

\section{Detection and Classification using RBFNN}

This section discusses about the simulation of combined wavelet transformation with RBFNN for classification of 5 types of power quality problems. Here Amplitude, Slope, Energy, Standard Deviation, Variance, Skewness, Kurtosis, Shannon Entropy, Log Energy Entropy and Norm Entropy were used as inputs to the RBFNN. Input signals for training is been selected by random signal at a time.
The training is set for learning rate 0.01 and target error 0.001. Each network is trained with 30 input data of each class and 100 data of each class are considered for testing. Weights is updated in each and every iteration. In this way new training input is given to the network. The randomly selected signal from 100 signals of each power quality problem is used to test RBFNN. To evaluate the performance of RBFNN, their results are compared. The classification result during testing is shown in Table 6 . The overall classification accuracy of RBFNN is $96.8 \%$.

\section{Comparison of Proposed Work with Real Time Data}

In this section, to check the proposed networks potential, less number of events that is voltage sag, swell and under voltage and transients where used with 10 orientations, with different indices. The generated signals features were used for training and tested with practical data. To test the proposed work, data of [12] mainly for ideal signal (230 vrms and $50 \mathrm{~Hz}$ ), Sag with ( $40 \%$ and $20 \mathrm{~ms})$, under voltage $(40 \%$ and $1 \mathrm{~ms})$ and swell $(20 \%$ and $60 \mathrm{~ms})$ were taken and then from them the features were extracted and given as input to the proposed trained FL system.

In similar way to test the potential of the proposed network the data of [23] mainly transient and sag were taken which is an experimental setup monitored at the point of common coupling, composed of a transformer bank in delta-wye of $350 \mathrm{VA}$, a capacitor bank of $77 \mu \mathrm{F}$ and two motors of 1 and $2 \mathrm{hp}(746 \mathrm{~W})$ respectively with data acquisition system with an low pass Butterworth antialiasing filter. The comparison results were shown in Table 3.

From the Table 3 it is inferred that the proposed fuzzy technique has the potential to deal with any type of data to produce better detection and classification rate, since the extracted membership function has a wide deviation which covers all categories of disturbances. Whereas 10 numbers of orientation may not be adequate in real cases for detection and classifications of PQ events. 
Table 3. Comparison of Proposed Fuzzy C-Means with Other works Practical Data

\begin{tabular}{|c|c|c|c|c|}
\hline \multirow{2}{*}{$\begin{array}{l}\text { Test } \\
\text { Signals }\end{array}$} & \multirow{2}{*}{$\begin{array}{l}\text { Disturba } \\
\text { nces }\end{array}$} & \multicolumn{3}{|c|}{ Classification rate $\%$} \\
\hline & & $\begin{array}{l}\text { RBF } \\
\mathrm{NN}\end{array}$ & $\begin{array}{l}\text { FUZZ } \\
Y\end{array}$ & $\begin{array}{l}\text { FUZZY } \\
\text { C-Means } \\
\text { Clusterin } \\
\mathrm{g}\end{array}$ \\
\hline \multirow{4}{*}{$\begin{array}{l}\text { Simulat } \\
\text { ed } \\
\text { Signals } \\
\text { using } \\
\text { MATL } \\
\text { AB }\end{array}$} & Sag & 97 & 97 & 98 \\
\hline & Swell & 94 & 95 & 97 \\
\hline & $\begin{array}{l}\text { Under } \\
\text { voltage }\end{array}$ & 96 & 98 & 98 \\
\hline & $\begin{array}{l}\text { Transient } \\
\mathrm{s}\end{array}$ & 97 & 97 & 99 \\
\hline \multirow{4}{*}{$\begin{array}{l}\text { InigoM } \\
\text { oneder } \\
\text { oet } \\
\text { al.,200 } \\
7\end{array}$} & Sag & 97 & 98 & 98 \\
\hline & Swell & 96 & 95 & 97 \\
\hline & $\begin{array}{l}\text { Under } \\
\text { voltage }\end{array}$ & 95 & 97 & 96 \\
\hline & $\begin{array}{l}\text { Transient } \\
\mathrm{s}\end{array}$ & 96 & 98 & 98 \\
\hline \multirow[b]{2}{*}{$\begin{array}{l}\text { Martin } \\
\text { Valtierr } \\
\text { a - } \\
\text { Rodrig } \\
\text { ues et } \\
\text { al } 2014\end{array}$} & Sag & 98 & 97 & 97 \\
\hline & $\begin{array}{l}\text { Transient } \\
\mathrm{s}\end{array}$ & 95 & 97 & 99 \\
\hline
\end{tabular}

\section{Particle Swarm Optimization}

Population based optimization tool is the PSO. To get the optimal solution, every single solution'flies' over the solution space. To check how close they are optimal is evaluated by using a fitness function[19]. Usually some particles position will be better than the others, before reaching the stopping-criteria of algorithm. Particles use to changes their position in the space. These criteria can be stopped either by reaching a optimal state or ending the number of specific repetation. The position of each particle is changed on the base of knowing the neighboring particles.Usually all particles knows its best and former position and also best among all particles.The velocity vector of the particle is updated according to equation (11).

$\bar{v}_{l}(t+1)=w \bar{v}_{l}(t)+c_{1} r_{1}\left(\right.$ pbest $\left._{i}(t)-\bar{x}_{l}(t)\right)+$

$c_{2} r_{2}\left(\right.$ gbest $\left._{i}(t)-\bar{x}_{l}(t)\right)$

$w$ is the inertia weight factor and $\bar{v}_{l}(t)$ is the previous velocity of the particle, $\bar{v}_{l}(t+1)$ is the present velocity of the particle, $c_{1}$ and $c_{2}$ are weighting acceleration constants. Best is the best solution that the particle has achieved and indicates the tendency to replicate their corresponding past behaviors. gbest is the best solution that has achieved so far by the specific particle in the whole population, which indicates the tendency to follow the success of others by the particles. After the velocity updating the particles moves to new position $x_{i}(t+1)$ from $x_{i}(t)$ by equation (12).

$\bar{x}_{l}(t+1)=\bar{x}_{l}(t)+\bar{v}_{l}(t+1)$

If the goal of optimization is the minimization of the objective functions ' $f$ ' then both pbesti and gbesti are updated by equation (13) and (14) respectively.

$$
\begin{aligned}
& \text { pbest }_{<}(t+1)= \\
& \left\{\begin{array}{c}
\text { pbest }_{i}(t+1) \text { if } f\left(\text { pbest }_{i}(t+1) \leq f\left(\bar{x}_{l}(t+1)\right.\right. \\
\bar{x}_{l}(t+1) \text { if } f\left(\text { pbest }_{i}(t+1)>f\left(\bar{x}_{l}(t+1)\right.\right.
\end{array}\right.
\end{aligned}
$$

$$
\text { gbest }_{i}(t+1) \in \text { pbest }_{1}(t+1) \ldots \ldots \text { pbest }_{n}(t+
$$
1)

$$
\begin{aligned}
f\left(\text { pbest }_{i}(t+1)\right. & \\
& =\min \left\{f \left(\text { pbest }_{1}(t\right.\right. \\
& +1) \ldots \ldots f\left(\text { pbest }_{n}(t+1)\right)
\end{aligned}
$$

The inertia weight can be set according to the following equation (15)

$w=w_{\max }-\frac{w_{\max }-w_{\operatorname{mix}}}{I_{\max }} I$

Where $w_{\max }$ and $w_{\min }$ maximum and minimum weight. 


\section{PSO-Implementation in Fuzzy C- Means Clustering}

PSO is used to improve the fuzzy C-means clustering even more in terms of classification accuracy. Suitable selection of centers will give the better clustering performance which is the main parameters to be optimized and given more priority. Initially the centers are estimated with the help of fuzzy c-means clustering which is used as an initial parameters for PSO and then in PSO all the data points wheretaken as input features then two initial centers were chosen and updated with the help of Fuzzy C-means clustering with the updated centers and the classes for two different clusters were determined using equation (10). Then with the help of PSO considering the initial centers ' $n$ ' random center that is particle where generated and from them new center and new classes for two different cluster where updated and distance between data points and each center were calculated and stored the Euclidean distance ina matrix M.And then the minimum distance where calculated with the relation (16).

$\mathrm{dmin}=\min (\mathrm{M})$

From the minimum distance the corresponding optimal PSO center were founded and done for ' $\mathrm{K}$ ' number of iteration to get the optimized PSOcenter. In this way PSO is used in Fuzzy CmeansClustering for all the data points and obtained different clusters, and shown in Figure.5 for transients and swell. The percentage accuracy using this technique is shown in the Table 6.From the table it is inferred that Fuzzy C-means clustering groups the events in a better way than a conventional technique.

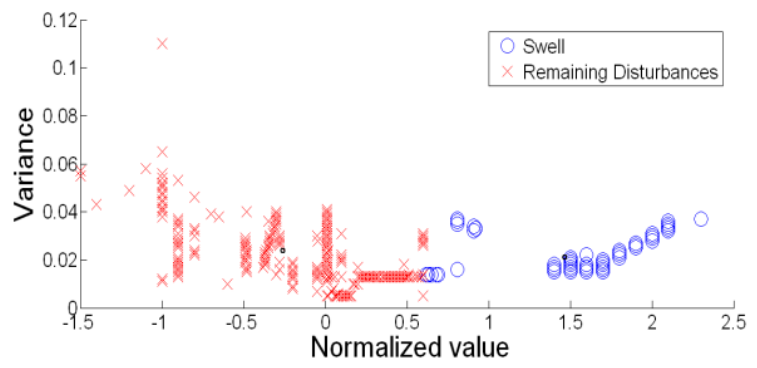

Fig. 4 Classification of the whole data set in to clusters of swell and Remaining Disturbances

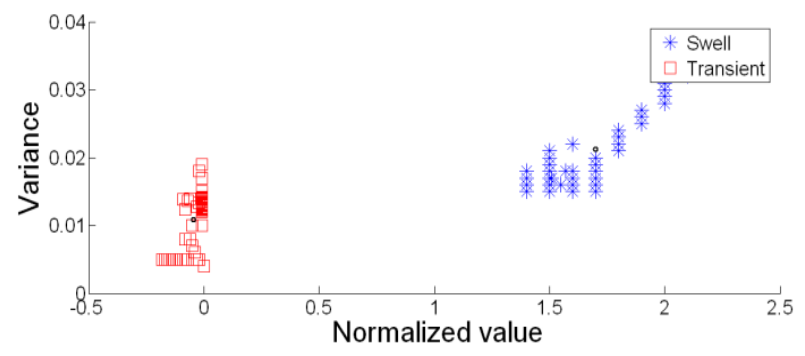

Fig. 5 Classification of the whole data set in to clusters of Transient and Swell

\section{Result and Discussion based on Features}

In any expert system approach, the main difficulty is that, if the number of input variables increases, system will take more time to respond. Hence, selection of features and number of features is necessary to any expert system approach for the real time problems. The performance of the network can be improved in terms of accuracy and convergence speed by reducing the number of features. This work proposes Wavelet Transform-MRA based feature selection technique. The input features are selected based on the values of Amplitude, Slope, Standard Deviation, Skewness, Kurtosis,Shannon Entropy, LogEnergy Entropy and Norm Entropy of both detail and approximate coefficients of the signals. In different resolution levels, the energy of the wavelet coefficient varies.

Energy of the low frequency signals and high frequency signals is distributed in approximation coefficients and in detail coefficients. Since, in real time the waveforms have higher frequency components, it is more desirable to use detailed coefficient energies. The performance of the proposed wavelet is compared based on the feature and number of features used for various classifier networks, In which first the analysis where done with three features such as Amplitude, Slope and Energy. Thenwith feature that is for Amplitude, slope, energy, standard deviation, Variance and then with all the 10 proposed features the results were shown in Table 4.

From the Table 4 it is inferred that network with less number of features give high classification, especially in Fuzzy C-means Clustering.

\section{Result and Discussion based on Features}

So it is desirable to use less features to get better classification which is very much needed in real time online applications. The performance of the proposed wavelet based features were compared 
with other works done with features extracted using S-Transforms ${ }^{16,24}$ andfeature extracted using STransforms and T-Transformas ${ }^{6}$.The performance results were shown in Table 5.From the Table 4 and Table 5 it is inferred that the proposed wavelet based feature selection gives better classification rate with lesser number of features when compared with other works.

Table 4.Comparison of Proposed Fuzzy C-Means on Number of Features with Other Technique

\begin{tabular}{|c|c|c|}
\hline $\begin{array}{c}\text { Number } \\
\text { of } \\
\text { Features } \\
\text { used }\end{array}$ & Classifier & $\begin{array}{c}\text { Classification } \\
\text { rate } \%\end{array}$ \\
\hline \multirow{3}{*}{3} & RBFNN & 96.80 \\
\hline & FUZZY & 97.40 \\
\hline & $\begin{array}{l}\text { FUZZY C-Means } \\
\text { Clustering }\end{array}$ & 98.40 \\
\hline \multirow{3}{*}{5} & RBFNN & 95.85 \\
\hline & FUZZY & 96.15 \\
\hline & $\begin{array}{l}\text { FUZZY C-Means } \\
\text { Clustering }\end{array}$ & 97.50 \\
\hline \multirow{3}{*}{10} & RBFNN & 95.95 \\
\hline & FUZZY & 96.85 \\
\hline & $\begin{array}{l}\text { FUZZY C-Means } \\
\text { Clustering }\end{array}$ & 97.30 \\
\hline
\end{tabular}

\section{Performances under Noisy Condition}

The inputs considered so far are noise free. However, the signals in the real system will always have noise. In order to test the robustness of Fuzzy c-means Clustering and Fuzzy c-means Clustering PSO, the white noise, which has random normal distribution, is added to normal signal to test the performance of
Table 5.Comparison of Proposed Fuzzy C-Means on Number of Features with Other Work

\begin{tabular}{|c|c|c|c|}
\hline Features & $\begin{array}{l}\text { Number } \\
\text { of } \\
\text { features } \\
\text { used }\end{array}$ & $\begin{array}{l}\text { Classifie } \\
\text { r }\end{array}$ & $\begin{array}{l}\text { Classific } \\
\text { ation } \\
\text { rate } \%\end{array}$ \\
\hline \multirow{2}{*}{$\begin{array}{l}\text { Features } \\
\text { Extracted using } \\
\text { S-Transforms } \\
\text { ( S. Mishra et } \\
\text { al } 2008 \text { ) }\end{array}$} & 4 & PNN & 97.4 \\
\hline & 3 & PNN & 95.91 \\
\hline \multirow{3}{*}{$\begin{array}{l}\text { Feature } \\
\text { Extracted using } \\
\text { S-Transforms } \\
\text { and T- } \\
\text { Transformas } \\
\text { (Chun-Yao Lee } \\
\text { and Yi-Xng } \\
\text { shen.2011) }\end{array}$} & \multirow{3}{*}{5} & APNN & 96.3 \\
\hline & & MLP & 98.1 \\
\hline & & K-NN & 96.0 \\
\hline \multirow[b]{2}{*}{$\begin{array}{l}\text { Features } \\
\text { Extracted using } \\
\text { S-Transforms } \\
\text { ( } \\
\text { PrakashK. Raye } \\
\text { t al 2013) }\end{array}$} & \multirow[b]{2}{*}{10} & MPNN & 96.66 \\
\hline & & SVM & 98.33 \\
\hline \multirow{3}{*}{$\begin{array}{l}\text { Features } \\
\text { Extracted using } \\
\text { Wavelet } \\
\text { Transforms } \\
\text { Proposed }\end{array}$} & \multirow[t]{3}{*}{3} & FUZZY & 97.40 \\
\hline & & $\begin{array}{l}\text { FUZZY } \\
\text { C- } \\
\text { Means } \\
\text { Clusteri } \\
\text { ng } \\
\end{array}$ & 98.40 \\
\hline & & $\begin{array}{l}\text { FUZZY } \\
\text { C- } \\
\text { Means } \\
\text { Clusteri } \\
\text { ng -PSO }\end{array}$ & 98.80 \\
\hline
\end{tabular}


Table 6. Performance Comparison

\begin{tabular}{|c|c|c|c|c|c|c|c|c|c|}
\hline \multirow{3}{*}{$\begin{array}{l}\text { Power } \\
\text { Quality } \\
\text { Events }\end{array}$} & \multicolumn{9}{|c|}{ Comparison of classification rate in $\%$} \\
\hline & \multicolumn{4}{|c|}{ References } & \multicolumn{5}{|c|}{ Proposed } \\
\hline & $\begin{array}{l}\text { Martin } \\
\text { Valtierra- } \\
\text { Rodriguez } \\
\text { et al.,2014 }\end{array}$ & $\begin{array}{l}\text { Prakash } \\
\text { K. Ray } \\
\text { et } \\
\text { al.,201 } \\
3\end{array}$ & $\begin{array}{l}\text { Mishra } \\
\text { et } \\
\text { al.,200 } \\
8\end{array}$ & $\begin{array}{l}\text { InigoMo } \\
\text { nederoet } \\
\text { al.,2007 }\end{array}$ & $\begin{array}{l}\text { RBFN } \\
\mathrm{N}\end{array}$ & FL & $\begin{array}{l}\text { FUZZY } \\
\text { C- } \\
\text { Means }\end{array}$ & $\begin{array}{l}\text { FUZZY } \\
\text { C- } \\
\text { Means- } \\
\text { PSO }\end{array}$ & $\begin{array}{l}\text { FUZZY } \\
\text { C- } \\
\text { Means- } \\
\text { PSO (20 } \\
\text { dB } \\
\text { Noise) }\end{array}$ \\
\hline $\mathrm{C} 1$ & 100 & -- & 100 & 90 & 100 & 100 & 100 & 100 & 98 \\
\hline $\mathrm{C} 2$ & 100 & 100 & 95 & 90 & 97 & 97 & 98 & 99 & 93 \\
\hline $\mathrm{C} 3$ & 100 & 97 & 91 & 70 & 94 & 95 & 97 & 97 & 95 \\
\hline $\mathrm{C} 4$ & -- & -- & 99 & -- & 96 & 98 & 98 & 98 & 96 \\
\hline C5 & 98 & -- & 100 & -- & 97 & 97 & 99 & 100 & 94 \\
\hline
\end{tabular}

Fuzzy c-means Clustering -PSO under noisy environment. The signal to noise ratio (SNR) 20, 30 and $40 \mathrm{db}$ noise level where used for detection and classification. The test results are depicted in Table VI. As seen from the simulation results, wavelet transformation with Fuzzy c-means Clustering -PSO is able to detect and classify the power quality problems accurately even with noise. The classification accuracy of the FL is improved, just by determining the ranges of the feature of the membership function for each rule to identify each disturbance specifically by PSO algorithm as discussed earlier. The performance of Fuzzy cmeans Clustering -PSO is compared with FL for both with and without noise and with other works done with features extracted using S-Transforms [16], [24] and feature extracted using their features [12],[ 22] which is shown in Table 6. From the Table 6 it is inferred that proposed method stands an evident that it can be implemented in any online application.

\section{Conclusion}

In this paper, the application of wavelet transforms combined with RBFNN, Fuzzy Logic technique and Fuzzy c-means clustering to detect and classify various PQ disturbances is presented. A numerical simulation is conducted to exhibit the properties of WT-based MRA. The feature extracted by wavelet is used as inputs to RBFNN and for to create membership function in fuzzy Logic and features to group various events using Fuzzy c-means Clustering detection and classification. The classification accuracy of the Fuzzy c-means clusteringis improved, just by determining the ranges of the feature for membership function for each rule to identify each disturbance specifically with the help of PSO. The performance of Fuzzycmeans Clustering-PSO stands an evident that it can be implemented in any online application and may help to take appropriate measures while facing the power quality problems. 


\section{References}

[1] W.R.A.Ibrahim and M.M.Morcos,"Artifical intelligence and advanced mathematical for power quality application, $A$ survey", IEEE Trans. Power Del.,Vol.17,No.2,pp.668-673. 2002.

[2] R.A.Flores, "State of the art in the classification of power quality events, an overview"in Proc.10 Int. Conf. Harmonics Quality of Power,Vol.1,pp.17-20, 2002.

[3] Y.H.Gu and M.H.J.Bollen,"Time-frequency and time -scale domain analysis of voltage disturbances",IEEE

Trans.Power Del.Vol.15,No.4,pp.1279-1284, 2002

[4] A.M.Gorgoom,N.Ertugrul,and W.L Soong, "A comparative study on effective signal processing tools for power quality monitoring,"in Proc.11thEur. Conf.Power

[5] B.K.Panigrahi and V.R.Pandi, “ Optimal feature selection for classification of power quality disturbances using waveletpacketbasedfuzzyK-nearest algorithim”, Inst.Eng, Technol.Gen, Transm.Distrib., Vol.3,No.3 pp.296-306, 2009.

[6] Chun-Yao Lee and Yi-Xing shen,"Optimal Feature Selection for Power Quality DisturbancesClassification",IEEETrans.Power Del. Vol.26,No.4,pp.2342-2351,2000.

[7] W.EdwardReid,"Power Quality Issues Standards and Guidelines", IEEE Trans.IndustryApplications, Vol.32,No.3,pp.62 $5632,1996$.

[8] .Elmitwally,S.Farghal,M.Kandil,S.Abdelkader and M.Elkateb,proposed,"wavelet-nerofuzzy coimbined system for power quality vioilation detection and diagnsis" Pros.Inst. Elect.Eng.,Gen,Transm.Distrib.,Vol.148,No.1 ,pp.15-20,2001.

[9] Mallat,S.G,“A Theory for Multiresolution Signal Decomposition, The Wavelet representation,'IEEETrans.Pattern
Recognition
and
Machine

Intelligence,Vol.11pp.674 -693,1989
[10] T.Mcconaghy, H.Lung, E.Bose; V.Vardan, "Classifcaton of Audio radar signals using Radial Basis Function Neural Networks,'IEEETrans.Inst.and Measurements,Vol.52,No.6,pp.1771-1779, 2003.

[11] Chia-Hung Lina and Chia-Hao Wang, "Adaptive Wavelet Networks for Power Quality Detection and Discrimination in a Power system", IEEE Trans.PowerDel. Vol.21,No.3,pp.1106-1113,2006

[12] InigoMonedero,Carlos Leon,JorgeRopero; Antonio Garcia and Jose Manuel Elena,“ Classification of Electrical Disturbances in Real Time using NeuralNetworks," IEEE Trans. Power Del.,Vol.22,No.2,pp. 1288$1296,2007$.

[13] Masoum,M.A.S,Jamali,S and Ghaftarzadeh, $\mathrm{N}$,"Detection and Classification of power quality disturbances using discrete wavelet transform and wavelet network," IET science,measurements \& technology.,Vol.4,pp.193-205,2010.

[14] Z.L.Gaing,"Wavelet-Based neural networks for power disturbance recognition and classification,"IEEE Trans.Power Del. Vol.19,No.4,pp. 1560 -1568,2004.

[15] A.Garcia-Perez and E. CabalYepez,"Techniques and methodologies for power quality analysis and disturbances classification in power systems A review", IET Gener. Transm.Distrib.Vol. 5,No.4,pp.519$529,2011$.

[16] S.Mishra,C.N.Bhende and B.K.Panigrahi, "Detection and Classification of Power Quality Disturbances using S-Transform and Probabilstic Neural Networks," IEEE Trans .Power Del.,Vol.23,No.1,pp.280-286,2008.

[17] P.K.Dash, S.Mishra, M.M.A.Salama and a.c.Liew, "Classification of Power System Disturbances Using a Fuzzy Expert System and a Fourier Linear Combiner," IEEE Trans.Power Del.,Vol.15,No.2,2000.

[18] X.Hu,Y.Shi and R.Eberhart, "Recent advances in Particle Swarm Optimization", Proceedings of the congress onEvolutionary 
Computation, Portland, OR, USA, Vol. 1,pp.90-97,2004.

[19] R.C.Eberhart and Y. Shi, "Particle swarm Optimization Developments, Applications and Resources", Proceedngs of the 2001 Congress on Evolutionary Computation, Vol.1,pp.81-86,2001.

[20] K.G Narendra, V.K.Stood, K.Khrasani and R.Patel, "Application of radial basis function (RBF) Neural Network for Fault diagnsis in a HVDC system",IEEETrans.OnPower system,Vol.13,No.1,pp. 117-183,1998

[21] M MamumBin Ibnereaz, FlorenceChong, MohdshahimanSulaiman, Faisal MohdYasin "Expert System for Power Quality Disturbance Classifer" IEEE Trans. Power Del, Vol.22,No.3, 2007.

[22] Martin Valtierra-Rodriguez,Rene de jesus Romero-Troneoso,Rogue Alfredo Oscornioios and Arturo Garcia-Perez,"Detection and Classification of single and combined power quality disturbance using Neural networks,'IEEETrans.on.Industrial electronics.,Vol.1,No.5,pp.2473- 2482,2014.

[23] Birenra Biswal, P.k.Dash and B.K.Panigrahi, “ Power Quality Disturbance Classification using Fuzzy C-Means algorithm and Adaptive Particle Swarm Optimization,"IEEE Trans. Power Del., Vol.56, No. 1, pp.212-220,2009.

[24]PrakashK.Ray, Soumy a R.Mohanty and NandKishor, "Classification of Power Quality Disturbances Due to Environmental Characteristics in Detection Generation Systems,"IEEE Trans.on Sustainable energy., Vol.4,No.2,pp.302-313,2013.

\section{Creative Commons Attribution License 4.0} (Attribution 4.0 International, CC BY 4.0)

This article is published under the terms of the Creative Commons Attribution License 4.0

https://creativecommons.org/licenses/by/4.0/deed.en_US 